Pacific Journal of Mathematics

DIVISORS OF POLYNOMIALS AND POWER SERIES WITH

and ERNSt Gabon Straus 


\title{
DIVISORS OF POLYNOMIALS AND POWER SERIES WITH POSITIVE COEFFICIENTS
}

\author{
T. S. Motzkin and E. G. Straus
}

\begin{abstract}
A polynomial $P\left(x_{1}, \cdots, x_{n}\right)$ is positive if its coefficients are $\geqq 0$ and not all 0 , copositive if it is $>0$ whenever the variables are $\geqq 0$ and not all 0 . Evidently (if needed, after multiplication by -1$)$ every real divisor $Q$ of a positive polynomial $P$ with $P(0) \neq 0$ is copositive. Conversely, every real copositive polynomial $Q$ with copositive highest and copositive lowest homogeneous part is a divisor of a positive polynomial $P$, and $P / Q$ may be chosen (1) as a product of positive 1-variable polynomials and positive homogeneous polynomials; or, alternatively, $(2)$ positive and so that all terms of $P$ from its lowest up to its highest degree are positive; or, if $n=1,(3)$ so that $P$ has no more nonzero terms than $Q$; or, for $n=1$ and quadratic $Q$, (4) as a power of a positive linear function, or (5) so that (1) and (3) hold. For power series in a multidisk analogous results hold, for $n=1$ partly depending on the finiteness of the number of complex zeros in the disk.
\end{abstract}

If $P(x)$ is a positive polynomial, that is

$$
P(x)=P\left(x_{1}, \cdots, x_{n}\right)=\sum_{\mu_{n}=0}^{m_{n}} \cdots \sum_{\mu_{1}=0}^{m_{1}} c_{\mu_{1} \ldots \mu_{n}} x_{1}^{\mu_{1}} \cdots x_{n}^{\mu_{n}}
$$

with $c_{\mu_{1} \ldots \mu_{n}} \geqq 0, \quad c_{0 \ldots 0}>0$, then $P(x) \neq 0$ for all $x$ in the nonnegative orthant $x_{\mu} \geqq 0 ; \mu=1, \cdots, n$. This property is obviously inherited by all polynomial divisors of $P$. In this note we examine to what extent, conversely, every polynomial without real nonnegative zeros is the divisor of a positive polynomial and investigate the type of multipliers that yield positive products as well as the type of positive products obtainable.

In $\S 2$ we consider polynomials of one variable. In $\S 3$ we generalize these results to functions of an arbitrary number of variables. In $\$ 4$ we consider generalizations to power series.

2. Polynomials of one variable without nonnegative zeros. We may clearly restrict attention to polynomials with real coefficients since the 1.c.m. of $P(x)$ and $\bar{P}(x)$ has a nonnegative zero if and only if $P(x)$ does. Factoring the (monic) real polynomial $P(x)$ over the reals we may get some monic first degree polynomials, which by hypothesis have positive coefficients, and some second degree factors

$$
Q(x)=x^{2}-(2 r \cos \theta) x+r^{2}
$$

corresponding to pairs of complex conjugate zeros $r e^{ \pm i \theta}, 0<\theta<\pi$. 
Theorem 2.1. Let $n$ be the integer such that $(n-1) \theta<\pi \leqq n \theta$; then a positive polynomial divisible by $Q(x)=x^{2}-(2 \cos \theta) x+r^{2}$ must be of degree $d \geqq n$. If $n \theta=\pi$ then there exists a unique monic polynomial $P(x)=x^{n}+r^{n}$ divisible by $Q(x)$. If $n \theta>\pi$ then for each $m, 0<m<n$, there exists a unique positive monic trinomial

$$
P_{m}(x)=x^{n}-\frac{\sin n \theta}{\sin m \theta} r^{n-m} x^{m}+\frac{\sin (n-m) \theta}{\sin m \theta} r^{n}
$$

divisible by $Q(x)$. The polynomials $P_{m}(x)$ are the vertices of the simplex of positive multiples of $Q(x)$ in the space of all monic polynomials of degree $n$.

If $P(x)$ is a positive monic multiple of $Q(x)$ and $\operatorname{deg} P=n$ then $P(x) / Q(x)$ is monic with all coefficients $>0$.

Proof. Since the points $1, r e^{i \theta}, \cdots, r^{n-1} e^{i(n-1) \theta}$ all lie in the angle $0 \leqq \arg z \leqq(n-1) \theta<\pi$, any linear combination with nonnegative coefficients lies in this angle; it can therefore be zero only if all the coefficients are zero. Hence if $P(x)$ is monic positive and $\operatorname{deg} P<n$ then $P\left(r e^{i \theta}\right) \neq 0$ so that $P$ is not a multiple of $Q$.

If $n \theta=\pi$ then the points $1, r e^{i \theta}, \cdots, r^{n-1} e^{i(n-1) \theta}, r^{n} e^{i n \theta}$ all lie in the closed half-plane $0 \leqq \arg z \leqq \pi$ and hence the only vanishing linear combination with positive coefficients is a multiple of $r^{n} \cdot 1+1 \cdot r^{n} e^{2 n \theta}$, so that the only monic positive multiple of $Q$ of degree $n$ is $P(x)=x^{n}+r^{n}$. The ratio

$$
\begin{aligned}
\frac{P(x)}{Q(x)}=x^{n-2} & +\frac{\sin 2 \theta}{\sin \theta} r x^{n-3} \\
& +\frac{\sin 3 \theta}{\sin \theta} r^{2} x^{n-4}+\cdots+\frac{\sin (n-1) \theta}{\sin \theta} r^{n-2}
\end{aligned}
$$

has strictly positive coefficients.

If $n \theta>\pi$ then the point $-r^{n} e^{i n \theta}$ lies in the open angle $0<\arg z<m \theta$ for each $m=1, \cdots, n-1$. We therefore have a unique linear combination with positive coefficients of 1 and $r^{m} e^{i m \theta}$ which yields $-r^{n} e^{i n \theta}$, namely

$$
-\frac{\sin n \theta}{\sin m \theta} r^{n-m}\left(r^{m} e^{i m \theta}\right)+\frac{\sin (n-m) \theta}{\sin m \theta}=-r^{n} e^{i n \theta},
$$

which shows that (2.2) yields the only positive monic trinomial involving $1, x^{m}$ and $x^{n}$ which is divisible by $Q$. The ratio

$$
\begin{aligned}
\frac{P_{m}(x)}{Q(x)}= & x^{n-2}+\frac{\sin 2 \theta}{\sin \theta} r x^{n-3}+\cdots+\frac{\sin (n-1) \theta}{\sin \theta} r^{n-2} \\
& -\frac{\sin n \theta}{\sin m \theta} r^{n-m}\left(x^{m-2}+\frac{\sin 2 \theta}{\sin \theta} r x^{m-3}+\cdots+\frac{\sin (m-1) \theta}{\sin \theta} r^{m-2}\right)
\end{aligned}
$$


has all coefficients $>0$.

It is clear that any combination $\sum_{m=1}^{n-1} \lambda_{m} P_{n}(x)$ with $\lambda_{m} \geqq 0, \sum \lambda_{m}=1$ is a monic positive multiple of $Q$ of degree $n$. Conversely, if

$$
P=c_{0} x^{n}+c_{1} x^{n-1}+\cdots c_{n}, \quad c_{0}>0,
$$

is a positive multiple of $Q$ and $P \neq c_{0} P_{m}$, then so is

$$
P+\frac{c_{m} \sin m \theta}{r^{n-m} \sin n \theta} P_{m}
$$

for each $m=1, \cdots, n-1$. For, otherwise, let $\lambda_{m}$ be the maximal number for which $P-\lambda_{m} P_{m}$ has nonnegative coefficients. Then either $\lambda_{m}=c_{0}$ and $\operatorname{deg}\left(P-\lambda_{m} P_{m}\right)<n$, a contradiction; or

$$
\lambda_{m}=\frac{c_{n} r^{-n} \sin m \theta}{\sin (n-m) \theta}
$$

and $P-\lambda_{m} P_{m}$ is divisible by $x$. But then $\left(P-\lambda_{m} P_{m}\right) / x^{k}$ is a positive multiple of $Q$ of degree $n-k$ for some $k \geqq 1$, a contradiction. Repeating this argument we get

$$
P=-\sum \frac{c_{m} \sin \theta m}{r^{m-m} \sin n \theta} P_{m} .
$$

CoRollary 2.3. If $N \theta>\pi$ then the set $\mathscr{R}_{N}$ of positive polynomials $R(x)$ of degree $N-2$ such that $R(x) Q(x)$ is positive has a nonempty interior in the space of all polynomials of degree at most $N-2$. In particular, there exists an $R(x)$ with positive integral coefficients so that all coefficients of $R(x) Q(x)$ are positive.

Proof. If we prove the corollary for one $N$ then it is proved for $N+1$ since the convex hull of $\mathscr{R}_{N}$ and $x \mathscr{R}_{N}$ is contained in $\mathscr{R}_{N+1}$ and has a nonempty interior in the space of all polynomials of degree at most $N-1$. Now if $n \theta>\pi$ in Theorem 2.1 then $\mathscr{R}_{n} Q$ was shown to consist of a nondegenerate simplex, so that $\mathscr{R}_{n}$ possesses interior points. If $n \theta=\pi$, then by the same argument as in the proof of Theorem 2.1 there exist trinomials

$$
\begin{aligned}
P_{m}=x^{n+1} & -\frac{\sin (n+1) \theta}{\sin m \theta} r^{n+1-m} x^{m} \\
& +\frac{\sin (n+1-m) \theta}{\sin m \theta} r^{n+1}
\end{aligned}
$$

for each $m=1, \cdots, n-1$ which are positive multiples of $Q$. Together with the two binomials $x^{n}+r^{n}$ and $x^{n+1}+r^{n} x$ these polynomials are the vertices of a nondegenerate simplex $\mathscr{R}_{n+1} Q$ so that $\mathscr{R}_{n+1}$ has interior 
points. Since the polynomials with rational coefficients are dense, it follows that $\mathscr{R}_{N}$ contains polynomials with positive rational coefficients. Multiplying by the denominator we therefore can find polynomials with positive integral coefficients in $\mathscr{R}_{N}$.

CoRollary 2.4. If a real polynomial $Q$ of degree $d$ has no zeros whose argument is less than $\pi / n$ then there exists a positive polynomial $R$ so that $R Q$ is positive and

$$
\operatorname{deg} R Q \leqq \max \{d, n d / 2\} .
$$

THEOREM 2.5. Let $Q(x)$ be a monic real polynomial of degree $d$ without nonnegative real zeros. Then among the positive multiples $P$ of $Q$ of minimal degree $n$ there are either at least $n-d+1 d i f$ ferent $(d+1)$-nomials or there is at least one $d$-nomial. (Here $k$ nomial means the sum of $k$ or fewer monomials.)

Proof. We know that $Q(x)$ has positive multiples. Let $m=n-d$. The set $\mathscr{R}$ of all $R(x)$ in the space of monic polynomials of degree $m$ is convex polyhedral, the intersection of $m+d$ halfspaces, determined by the linear inequalities which express the fact that the coefficients of $R Q$ are nonnegative. If $\mathscr{R}$ were unbounded then there would exist $R \in \mathscr{R}$ with arbitrarily large coefficients. Let $\|R\|$ denote the maximal absolute value of the coefficients. Then as $\|R\| \rightarrow \infty$ the polynomials $R /\|R\|$ have a convergent subsequence which converges to a nonzero polynomial $S$ with $\operatorname{deg} S<m$ so that $S Q$ is positive, a contradiction. If $\mathscr{R}$ is a bounded nondegenerate polyhedron then it has at least $m$ vertices $V_{\mu}$ so that each $V_{\mu} Q$ is positive of degree $n$ with at least $m$ coefficients equal to 0 . Thus each $V_{\mu} Q$ is a $(d+1)$ nomial. If $\mathscr{R}$ is a degenerate polyhedron then each vertex $V$ lies on at least $m+1$ bounding hyperplanes. Thus $V Q$ is a $d$-nomial.

It would be wrong to assume that the set $\mathscr{R}$ of multipliers $R(x)$ of minimal degree for which $R Q$ is positive must contain a positive polynomial. For example the cyclotomic polynomial $\Phi_{30}(x)$ divides $x^{15}+1$ but $\Phi_{30}$ has a quadratic factor $x^{2}-(2 \cos \pi / 15) x+1$ that divides no other monic positive polynomial of degree 15 or less, while

$$
\begin{aligned}
\frac{x^{15}+1}{\Phi_{30}(x)} & =\Phi_{2}(x) \Phi_{6}(x) \Phi_{10}(x) \\
& =x^{7}-x^{6}+x^{5}+x^{2}-x+1 .
\end{aligned}
$$

We conclude this section by showing that every real quadratic polynomial $Q$ without nonnegative real zeros, and hence every real polynomial without nonnegative real zeros, can be multiplied by powers of linear positive polynomials to yield positive products. 
THEOREM 2.6. If $\cos \theta \leqq m /(m+2)$ then

$$
P(x)=\left(x^{2}-(2 r \cos \theta) x+r^{2}\right)(x+r)^{m}
$$

is positive.

REMARK. For small $\theta$, the smallest admissible $m$ is asymptotically $4 n^{2} / \pi^{2}$, with $n$ as in Theorem 2.1.

Proof. We have

$$
\begin{aligned}
P^{(k)}(0)= & r^{m-k+2} m \cdots(m-k+3) \\
& \quad \cdot[k(k-1)-2 k(m-k+2) \cos \theta+(m-k+2)(m-k+1)] \\
\geqq & r^{m-k+2} \frac{m \cdots(m-k+3)}{m+2} \\
& \quad \cdot[k(k-1)(m+2)-2 k(m-k+2) m \\
& \quad+(m-k+2)(m-k+1)(m+2)] \\
= & r^{m-k+2} m(m-1) \cdots(m-k+3)(m-2 k+2)^{2}(m+1) /(m+2) \\
\geqq & 0 .
\end{aligned}
$$

By choosing $m$ odd we can make all $P^{(k)}(0)>0$.

CoRollary 2.7. A real polynomial $Q(x)$ without nonnegative real zeros can be multiplied by a polynomial $R(x)$ with only negative real zeros so that $R(x) Q(x)$ is positive. We can also require that all coefficients of $R(x) Q(x)$ are positive, and that the coefficients of $R(x)$ are integers.

3. Polynomials of several variables without zeros in the real nonnegative orthant. As in $\$ 2$ we may assume that we are dealing with real polynomials. Now assume that $Q\left(x_{1}, \cdots, x_{n}\right) \neq 0$ for all $x_{1} \geqq 0, \cdots, x_{n} \geqq 0$. Writing $x=r \xi$ where $r=\|x\|_{2}$ and $\xi=\left(\xi_{1}, \cdots, \xi_{n}\right)$ is a unit vector we can write

$$
Q(x)=Q_{0}+r Q_{1}(\xi)+\cdots+r^{d} Q_{d}(\xi)
$$

where $Q_{\delta}(x)$ is the homogeneous part of degree $\delta$ of $Q$. If $Q_{d}(x)$ has nonnegative zeros then it may be impossible to find nonzero homogeneous multiples of $Q_{d}(x)$ with nonnegative coefficients, so that the leading homogeneous part of any nonzero product $R(x) Q(x)$ has some negative coefficients. For example the polynomial $Q(x, y)=1+(x-y)^{2}$ has no real and hence no positive zeros, yet $(x-y)^{2} R(x, y)$ cannot have nonnegative coefficients for any nonzero polynomial $R(x, y)$. Thus, in order to generalize the results of $\S 2$ we must modify our hypothesis, by excluding "zeros at infinity" in the nonnegative orthant. 
THEOREM 3.2. If $Q(x)$ is a real polynomial without zeros in the nonnegative orthant and if the leading homogeneous part, $Q_{d}(x)$, has no zeros on the nonnegative orthant of the unit sphere, then there exist positive polynomials $R_{1}\left(x_{1}\right), R_{2}\left(x_{2}\right), \cdots, R_{n}\left(x_{n}\right)$ so that the homogeneous parts of $P(x)=R_{1}\left(x_{1}\right) \cdots R_{n}\left(x_{n}\right) Q(x)$ are positive on the nonnegative orthant of the unit sphere.

Proof. As a consequence of $\S 2$ we know that for each nonnegative unit vector $\xi$ there exists a positive polynomial $R_{\hat{\xi}}(r)$ so that $R_{\xi}(r) Q(r \xi)$ is a strictly positive polynomial in $r$. Hence $R_{\xi}(r) Q(r \eta)$ is a positive polynomial in $r$ for all unit vectors $\eta$ in a neighborhood $U_{\xi}$ of $\xi$. By the Heine-Borel theorem there exists a finite set of neighborhoods $U_{\xi^{1}}, \cdots, U_{\xi^{N}}$ which covers the nonnegative orthant of the unit sphere. Hence $R_{\xi^{1}}(r) \cdots R_{\xi^{N}}(r) Q(r \xi)$ is a positive polynomial in $r$ for all nonnegative vectors $\xi$. Now let

$$
R_{\nu}\left(x_{\nu}\right)=\Pi R_{\xi^{i}}\left(x / \xi_{\nu}^{2}\right)
$$

where the product is extended over all $\xi^{i}$ whose nonzero component of minimal index is the $\nu$-th component $\xi_{\nu}^{i}$. Then

$$
P(x)=R_{1}\left(x_{1}\right) \cdots R_{n}\left(x_{n}\right) Q(x)
$$

is a positive polynomial in $r$ for all $x$ in the nonnegative orthant. In other words the homogeneous parts of $P(x)$ are positive in the positive orthant.

We have thus reduced the problem to that of homogeneous divisors of positive polynomials.

LEMma 3.3. If $Q(x)$ is a homogeneous polynomial which is positive on the nonnegative orthant of the unit sphere then there exists a positive homogeneous polynomial $R(x)$ so that $R(x) Q(x)$ has positive coefficients.

Proof. We use induction on the number of variables, $n$. Consider the polynomial $Q^{*}\left(y_{1}, \cdots, y_{n-1}\right)=Q\left(y_{1}, \cdots, y_{n-1}, 1\right)$. Then by hypothesis $Q^{*}(y)>0$ for all $y$ in the nonnegative orthant and its leading homogeneous part $Q\left(y_{1}, \cdots, y_{n-1}, 0\right)$ is positive for all nonnegative unit vectors $y$. Thus by Theorem 3.2 there exists multiplier

so that

$$
R_{1}^{*}\left(y_{1}\right) \cdots R_{n-1}^{*}\left(y_{n-1}\right)
$$

$$
P^{*}(y)=R_{1}^{*}\left(y_{1}\right) \cdots R_{n-1}^{*}\left(y_{n-1}\right) Q^{*}(y)
$$

has homogeneous parts which are positive on the nonnegative orthant of the unit sphere in $y$-space. By the induction hypothesis each homo- 
geneous part $P_{\tilde{o}}^{*}$ of $P^{*}$ has a positive homogeneous multiplier $S_{o}^{*}(y)$ so that $P_{\hat{o}}^{*}(y) S_{\hat{o}}^{*}(y)$ has positive coefficients.

Thus $\left(I_{\hat{o}} S_{\hat{o}}^{*}(y)\right) P^{*}(y)$ has positive coefficients. The polynomial

$$
x_{n}^{D} \prod_{\bar{o}} S_{\hat{o}}^{*}\left(\frac{x_{1}}{x_{n}}, \cdots, \frac{x_{n-1}}{x_{n}}\right) P *\left(\frac{x_{1}}{x_{n}}, \cdots, \frac{x_{n-1}}{x_{n}}\right)
$$

where $D=\operatorname{deg}\left[\left(\Pi_{i} S_{\hat{o}}^{*}(y)\right) P^{*}(y)\right]$, has now the desired property.

Combining Theorem 3.2 and Lemma 3.3 we get

THEOREM 3.4. For every real polynomial Q(x) which is positive in the nonnegative orthant and whose leading homogeneous part is positive on the nonnegative orthant of the unit sphere, there exist positive polynomials $R_{1}\left(x_{1}\right), \cdots, R_{n}\left(x_{n}\right)$ and positive homogeneous polynomials $S_{1}(x), \cdots, S_{N}(x)$ so that the product

$$
P(x)=R_{1}\left(x_{1}\right) \cdots R_{n}\left(x_{n}\right) S_{1}(x) \cdots S_{N}(x) Q(x)
$$

has nonnegative coefficients.

If $Q(0)=0$ is allowed then we must make a similar assumption for the "trailing" (lowest-degree) homogeneous part.

The polynomial $P(x)$ in (3.5) has homogeneous factors and therefore in general $P(0)=0$. However, assuming again $Q(0)>0$, it is possible to construct positive multiples $P(x)$ of $Q(x)$ so that any prescribed monomial has a positive coefficient or even so that all monomials whose degree is no higher than $\operatorname{deg} P$ have positive coefficients. To see this we proceed by induction on the number of variables, to find positive multipliers $R_{1}\left(x_{2}, \cdots, x_{n}\right), R_{2}\left(x_{1}, x_{3}, \cdots, x_{n}\right), \cdots, R_{n}\left(x_{1}, \cdots, x_{n-1}\right)$ so that $R_{2}(0, \cdots, 0)>0 ; \nu=1, \cdots, n$ and

$$
R_{\nu}\left(x_{1}, \cdots, x_{\nu-1}, x_{\nu+1}, \cdots, x_{n}\right) Q\left(x_{1}, \cdots, x_{\nu-1}, 0, x_{\nu+1}, \cdots, x_{n}\right)
$$

has nonnegative coefficients. If

$$
Q_{1}(x)=\left(\prod_{\nu=1}^{n} R_{\nu}(x)\right) Q(x)
$$

contains a monomial

$$
c_{\mu_{1} \cdots \mu_{n}} x_{1}^{\mu_{1}} \cdots x_{n}^{\mu_{n}}
$$

with $c_{i_{1} \ldots \mu_{n}}<0$ then $\mu_{\nu}>0$ for all $\nu=1, \cdots, n$. We consider the usual partial ordering of the $n$-tuples $\left(\mu_{1}, \cdots, \mu_{n}\right)$. If the polynomial $P(x)$ of (3.5) contains a monomial of lower index then there exists a positive multiple $P_{1}(x)$ of $P(x)$ so that $Q_{1}(x)+P_{1}(x)$ contains a positive multiple of $x_{1}^{\prime \prime} \cdots x_{n}^{\mu}$. The monomials with negative coefficients whose indices are not above those of monomials in $P(x)$ can be eliminated 
successively through multiplication by

where

$$
1+c_{\mu_{1} \cdots \mu_{n}}^{\prime} x_{1}^{\mu_{1}} \cdots x_{n}^{\mu_{n}}
$$

$$
c_{\mu_{1} \cdots \mu_{n}}^{\prime}>-Q_{1}(0) c_{\mu_{1} \cdots \mu_{n}} .
$$

To sum up we have the following.

THEOREM 3.6. For every polynomial $Q(x)$ which is nonzero in the nonnegative orthant and whose leading homogeneous part is nonzero on the nonnegative orthant of the unit sphere there exists a positive polynomial $R(x)$ so that $P(x)=R(x) Q(x)$ has positive coefficients for every monomial whose degree is no greater than $\operatorname{deg} P$.

If $Q(0)=0$ is allowed then $P(x)$ will only have positive coefficients for every monomial whose degree is no greater than $\operatorname{deg} P$ and no less than that of the trailing part of $P$.

We note that like Corollary 2.4 for polynomials of one variable, and Lemma 3.3 for homogeneous polynomials, but unlike Theorem 3.4, Theorem 3.6 implies that the class of divisors of polynomials with specified positivity or nonnegativity properties of their coefficients coincides with the class of polynomials which, together with their leading and their trailing part, have no zeros, other than 0 , in the nonnegative orthant.

In a manner analogous to that used in the proof of Theorem 2.5 we could get bounds on the minimal number of monomials in a positive multiple of $Q(\mathrm{x})$. The results are harder to state and we omit. them.

4. Divisors of positive power series.

THEOREM 4.1. Given a power series

$$
f(x)=1+\sum_{n=1}^{\infty} c_{n} x^{n}
$$

with radius of convergence $r(0<r \leqq \infty)$ and without zeros in the interval $[0, r)$, there exists a power series

$$
P(x)=1+\sum_{n=1}^{\infty} p_{n} x^{n}
$$

with $p_{n} \geqq 0 ; n=1,2, \cdots$ with radius of convergence $\geqq r$ so that $P(x) / f(x)$ is analytic for $|x|<r$.

Proof. Let the zeros of $f(x)$ be $x_{1}, x_{2}, \cdots$ with $x_{n}=r_{n} e^{i \theta_{n}}$ where $0<r_{1} \leqq r_{2} \leqq \cdots \leqq r_{n} \leqq \cdots<r$ and $0<\theta_{n}<2 \pi$. For each $\theta_{n}$ there 
exist arbitrarily large integers $N_{n}$ so that $N_{n} \theta_{n}$ is in the closed second quadrant, and $x_{n}$ satisfies the equation with nonnegative coefficients

$$
1-\left(2 \cos N_{n} \theta_{n}\right)\left(\frac{x}{r_{n}}\right)^{N_{n}}+\left(\frac{x}{r_{n}}\right)^{2 N_{n}}=0 .
$$

If we choose $N_{n}$ so large that $\left(r_{m} / r_{n}\right)^{N_{n}}<2^{-n}$ for all $r_{m}<r_{n}$ then the product

$$
P(x)=\Pi\left(1-\left(2 \cos N \theta_{n}\right)\left(\frac{x}{r_{n}}\right)^{N_{n}}+\left(\frac{x}{r_{n}}\right)^{2 N_{n}}\right)
$$

converges for all $|x|<r$ and has nonnegative coefficients. Since every zero of $f(x)$ in $|x|<r$ is a zero of no lower multiplicity of $P(x)$, it follows that $P / f$ is analytic in $|x|<r$.

The function $P(x)$ is a polynomial if $f$ has a finite number of zeros in $|x|<r$. Otherwise $P(x)$ has infinitely many zeros in $|x|<r$ and therefore its radius of convergence is $r$.

The construction of $P(x)$ does not permit us to say that $P / f$ can be chosen to be a positive power series and we have not been able to solve the following.

Problem 4.2. Assume the power series $f(x)$ of Theorem 4.1 is real. Is it possible to find a positive power series $g(x)$ with radius of convergence $r$ so that $f(x) g(x)$ has positive coefficients?

We can answer the problem in the affirmative for any radius of convergence $\rho<r$.

LEMMA 4.3. If $f(x) \neq 0$ in $|x|<r$ and $f(x)$ is a real power series, $f(0)=1$, then there exists a positive power series $g(x)$ converging for $|x|<r$ so that $f(x) g(x)$ has positive coefficients.

Proof. Under our hypothesis $\varphi(x)=\log f(x)$, normalized by $\varphi(0)=0$, is a real analytic function in $|x|<r$. Let $\varphi(x)=\sum_{n=1}^{\infty} b_{n} x^{n}$, then $g(x)=\exp \left(\sum_{n=1}^{\infty}\left|b_{n}\right| x^{n}\right)$ has the desired properties.

COROLlaRY 4.4. Let $f(x)$ be a real power series with radius of convergence $r$ and only a finite number of zeros in $|x|<r$ and no zeros in the interval $[0, r)$. If $f(0)=1$ then there exists a positive power series $g(x)$ converging for $|x|<r$ so that $f(x) g(x)$ has positive coefficients.

Proof. We can write $f(x)=Q(x) f_{1}(x)$ where $Q(x)$ is a polynomial and $f_{1}(x)$ satisfies the hypotheses of Lemma 4.3. Then by Corollary 
2.4 and Lemma 4.3 there exists a positive polynomial $R(x)$ and a positive power series $g_{1}(x)$ so that $Q(x) R(x)$ is positive and $f_{1}(x) g_{1}(x)$ is positive. Hence $g(x)=R(x) g_{1}(x)$ has the desired properties.

THEOREM 4.5. Let $f(x)$ be a real power series satisfying the hypotheses of Theorem 4.1. Then for every $\rho<r$ there exists a positive power series $g(x)$ with radius of convergence $\geqq \rho$ so that $f(x) g(x)$ is a positive power series.

Proof. In $|x|<\rho$ the function $f$ satisfies the hypotheses of Corollary 4.4 , with $r$ replaced by $\rho$.

In a manner analogous to that used in $\S 3$ we can now establish the following generalization of Theorem 4.5 to power series in several variables.

Theorem 4.6. Let

$$
f\left(x_{1}, \cdots, x_{n}\right)=1+\sum_{\mu_{1}+\cdots+\mu_{n}=1}^{\infty} c_{\mu_{1} \cdots \mu_{n}} x_{1}^{\mu_{1}} \cdots x_{n}^{\mu_{n}}
$$

be a real power series which converges for $\left|x_{\nu}\right|\left\langle r_{\nu} ; \nu=1, \cdots, n\right.$ and is positive for $0 \leqq x_{\nu}<r_{\nu} ; \nu=1, \cdots, n$. Then for every $\rho_{\nu}$ with $\rho_{\nu}<r_{\nu} ; v=1, \cdots, n$ there exist positive power series

$$
g_{\nu}\left(x_{\nu}\right)=1+\sum_{\mu=1}^{\infty} \gamma_{\nu \mu} x_{\nu}^{\mu} ; \quad r_{\nu \mu}>0
$$

with radius of convergence $\geqq \rho_{\nu}$ so that

$$
g_{\nu} f=\sum_{\mu=0}^{\infty} \varphi_{\nu / \mu}\left(x_{1}, \cdots, x_{\nu-1}, x_{\nu+1}, \cdots, x_{n}\right) x_{\nu}^{\mu} ; \quad \nu=1, \cdots, n
$$

where each $\varphi_{\nu \mu}$ is positive for

$$
0 \leqq x_{\lambda} \leqq \rho_{\lambda} ; \quad \lambda=1, \cdots, \nu-1, \nu+1, \cdots, n .
$$

Proof. For each vector $\xi=\left(\xi_{1}, \cdots, \xi_{\nu-1}, \xi_{\nu+1}, \cdots, \xi_{n}\right)$ with $0 \leqq \xi_{\lambda} \leqq$ $\rho_{\lambda}$, there exists by Theorem 4.5 a positive power series $P_{\xi}\left(x_{\nu}\right)$ with radius of convergence $\rho_{\nu}$ so that $P_{\xi}(0)=1$ and all the coefficients of $f\left(\xi_{1}, \cdots, \xi_{\nu-1}, x_{\nu}, \xi_{\nu+1}, \cdots, \cdots, \xi_{n}\right) P_{\xi}\left(x_{\nu}\right)$ (considered as a power series in $x_{\nu}$ ) are positive. If, as we may, we further multiply by $\left(1-x_{\nu} / \rho_{\nu}^{\prime}\right)^{-1}$ where $\rho_{\nu}<\rho_{\nu}^{\prime}<r_{\nu}$ then the power series

$$
\begin{aligned}
f\left(\xi_{1},\right. & \left.\cdots, \xi_{\nu-1}, x_{\nu}, \xi_{\nu+1}, \cdots, \xi_{n}\right) P_{\xi}\left(x_{\nu}\right)\left(1-x_{\nu} / \rho_{\nu}^{\prime}\right)^{-1} \\
& =\sum_{\mu=0}^{\infty} c_{\nu \mu}(\xi) x_{\nu}^{\mu}
\end{aligned}
$$


satisfies

$$
\frac{1}{\rho_{\nu}^{\prime \mu}}<c_{\nu \mu}(\xi)<\frac{M}{\rho_{\nu}^{\prime \mu}} ; \quad \mu=0,1,2, \cdots
$$

where $M$ is a positive constant independent of $\xi$. The upper bound is just an application of Cauchy's inequality. The functions $\rho_{\nu}^{\prime \mu} c_{\nu \mu}(\xi)$ are equicontinuous functions of $\xi$ for $0 \leqq \xi_{\lambda} \leqq \rho_{\xi}$; and hence there exists a neighborhood $U_{\xi}$ of $\xi$ so that

$$
f\left(\eta_{1}, \cdots, \eta_{\nu-1}, x_{\nu 1}, \eta_{\nu+1}, \cdots, \eta_{n}\right) P_{\xi}\left(x_{\nu}\right)\left(1-x_{\nu} / \rho_{\nu}^{\prime}\right)^{-1}
$$

has positive coefficients (as power series in $x_{\nu}$ ) for every $\eta \in U_{\xi}$. By the Heine-Borel theorem there is a finite set of neighborhoods $U_{\xi^{1}}, \cdots, U_{\xi^{N}}$ which covers the set

$$
0 \leqq \xi_{\lambda} \leqq \rho_{\lambda} ; \lambda=1, \cdots, \nu-1, \nu+1, \cdots, n
$$

so that

$$
f\left(x_{1}, \cdots, x_{n}\right) P_{\xi^{1}}\left(x_{\nu}\right) \cdots P_{\xi^{N}}\left(x_{\nu}\right)\left(1-x_{\nu} / \rho_{\nu}^{\prime}\right)^{-1}=f(x) g_{\nu}\left(x_{\nu}\right)
$$

satisfies the conditions of the theorem.

REMARK 4.7. Theorem 4.6 does not insure the existence of a multiple of $f(x)$ which is a power series with positive coefficients in $x_{1}, \cdots, x_{n}$. However the condition on the leading homogeneous part which we had to impose in $\S 3$ becomes vacuous for power series. For example the polynomial $1+(x-y)^{2}$, which cannot be the divisor of a positive polynomial, is the divisor of the positive entire function

$$
\begin{aligned}
f(x, y) & =\left[1+(x-y)^{2}\right] e^{(x+y)^{2}} \\
& =1+\sum_{n=1}^{\infty} \frac{n+1}{n !}\left(x^{2}-2 \frac{n-1}{n+1} x y+y^{2}\right)(x+y)^{2 n-2} .
\end{aligned}
$$

Now by a homogeneous version of Theorem 2.6 each of the polynomials

$$
\begin{aligned}
\left(x^{2}-\right. & \left.2 \frac{n-1}{n+1} x y+y^{2}\right)(x+y)^{2 n-2} \\
& =\left(x^{2}-2 \cos \theta x y+y^{2}\right)(x+y)^{m}
\end{aligned}
$$

has positive coefficients, since

$$
\cos \theta=\frac{n-1}{n+1}<\frac{n-1}{n}=\frac{2 n-2}{2 n}=\frac{m}{m+2} .
$$

Problem 4.8. Let $f\left(x_{1}, \cdots, x_{n}\right)$ be a power series which converges for $\left|x_{\nu}\right|<r_{\nu} ; \nu=1, \cdots, n$, so that $f(x)>0$ for $0 \leqq x_{\nu}<r_{\nu}$. 
Does there exist a power series $g(x)$ which converges for $\left|x_{\nu}\right|<r_{\nu}$; $\nu=1, \cdots, n$ so that $f g$ has positive coefficients? If so, can $g$ be chosen to have positive coefficients?

Received October 15, 1968. The preparation and research of this paper was partially supported by National Science Foundation Grants GP-7628 and GP-8622.

The synopsis is identical with the abstract in Notices Amer. Math. Soc., 15 (1968), 1027-1028.

University of CALifornia, LOS ANGeLes 


\section{PACIFIC JOURNAL OF MATHEMATICS}

\section{EDITORS}

H. ROYDEN

Stanford University

Stanford, California

\author{
R. R. Phelps \\ University of Washington \\ Seattle, Washington 98105
}

J. DugundJI

Department of Mathematics

University of Southern California

Los Angeles, California 90007

RICHARD ARENS

University of California

Los Angeles, California 90024

\section{ASSOCIATE EDITORS}

E. F. BECKenbaCH

B. H. NEUMANN

F. WOLF

K. YoSHIDA

\section{SUPPORTING INSTITUTIONS}

\author{
UNIVERSITY OF BRITISH COLUMBIA \\ CALIFORNIA INSTITUTE OF TECHNOLOGY \\ UNIVERSITY OF CALIFORNIA \\ MONTANA STATE UNIVERSITY \\ UNIVERSITY OF NEVADA \\ NEW MEXICO STATE UNIVERSITY \\ OREGON STATE UNIVERSITY \\ UNIVERSITY OF OREGON \\ OSAKA UNIVERSITY \\ UNIVERSITY OF SOUTHERN CALIFORNIA
}

\author{
STANFORD UNIVERSITY \\ UNIVERSITY OF TOKYO \\ UNIVERSITY OF UTAH \\ WASHINGTON STATE UNIVERSITY \\ UNIVERSITY OF WASHINGTON \\ $\stackrel{*}{*} \stackrel{*}{*} \stackrel{*}{*}$ AMERICAN MATHEMATICAL SOCIETY \\ CHEVRON RESEARCH CORPORATION \\ TRW SYSTEMS \\ NAVAL WEAPONS CENTER
}

The Supporting Institutions listed above contribute to the cost of publication of this Journal, but they are not owners or publishers and have no responsibility for its content or policies.

Mathematical papers intended for publication in the Pacific Journal of Mathematics should be in typed form or offset-reproduced, double spaced with large margins. Underline Greek letters in red, German in green, and script in blue. The first paragraph or two must be capable of being used separately as a synopsis of the entire paper. It should not contain references to the bibliography. Manuscripts, in duplicate if possible, may be sent to any one of the four editors. Please classify according to the scheme of Math. Rev. 36, 1539-1546. All other communications to the editors should be addressed to the managing editor, Richard Arens, University of California, Los Angeles, California, 90024.

50 reprints are provided free for each article; additional copies may be obtained at cost in multiples of 50 .

The Pacific Journal of Mathematics is published monthly. Effective with Volume 16 the price per volume (3 numbers) is $\$ 8.00$; single issues, $\$ 3.00$. Special price for current issues to individual faculty members of supporting institutions and to individual members of the American Mathematical Society: $\$ 4.00$ per volume; single issues $\$ 1.50$. Back numbers are available.

Subscriptions, orders for back numbers, and changes of address should be sent to Pacific Journal of Mathematics, 103 Highland Boulevard, Berkeley, California, 94708.

PUBLISHED BY PACIFIC JOURNAL OF MATHEMATICS, A NON-PROFIT CORPORATION

Printed at Kokusai Bunken Insatsusha (International Academic Printing Co., Ltd.), 7-17, Fujimi 2-chome, Chiyoda-ku, Tokyo, Japan. 


\section{Pacific Journal of Mathematics \\ Vol. 29, No. $3 \quad$ July, 1969}

Herbert James Alexander, Extending bounded holomorphic functions from certain subvarieties of a polydisc ...................... 485

Edward T. Cline, On an embedding property of generalized Carter

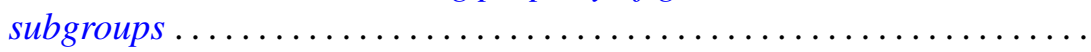

Roger Cuppens, On the decomposition of infinitely divisible characteristic functions with continuous Poisson spectrum. II ...............

William Richard Emerson, Translation kernels on discrete Abelian

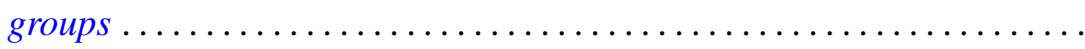

Robert William Gilmer, Jr., Power series rings over a Krull domain ....... 543

Julien O. Hennefeld, The Arens products and an imbedding theorem ...... 551

James Secord Howland, Embedded eigenvalues and virtual poles ........ 565

Bruce Ansgar Jensen, Infinite semigroups whose non-trivial homomorphs are all isomorphic .............................. 583

Michael Joseph Kascic, Jr., Polynomials in linear relations. II .......... 593

J. Gopala Krishna, Maximum term of a power series in one and several

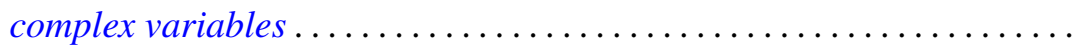

Renu Chakravarti Laskar, Eigenvalues of the adjacency matrix of cubic lattice graphs ...................................

Thomas Anthony Mc Cullough, Rational approximation on certain plane

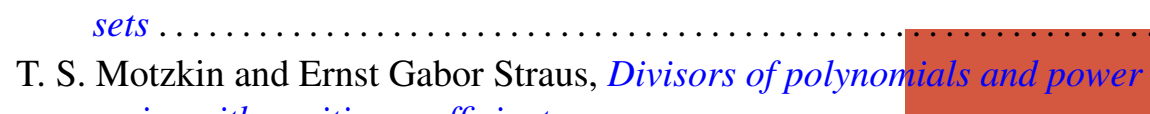
series with positive coefficients .

Graciano de Oliveira, Matrices with prescribed characteristic polynomial and a prescribed submatrix.

Graciano de Oliveira, Matrices with prescribed characteristic polynomial and a prescribed submatrix. II .

Donald Steven Passman, Exceptional 3/2-transitive permutation groups .................................

Grigorios Tsagas, A special deformation of the metric with no negative sectional curvature of a Riemannian space............

Joseph Zaks, Trivially extending decompositions of $E^{n}$ 